\title{
Psychological wellbeing in the English population during the COVID-19 pandemic: a series of cross-sectional surveys
}

Louise E Smith (0000-0002-1277-2564), ${ }^{1,2}$ PhD, Richard Amlôt (0000-0003-3481-6588),

PhD, Nicola T Fear (0000-0002-5792-2925), ${ }^{1,4}$ DPhil (Oxon), Susan Michie (0000-0003-

0063-6378), ${ }^{5}$ DPhil, G James Rubin (0000-0002-4440-0570), ${ }^{1,2}$ PhD, Henry WW Potts

(0000-0002-6200-8804), ${ }^{6} \mathrm{PhD}$.

1 King's College London, Institute of Psychiatry, Psychology and Neuroscience

2 NIHR Health Protection Research Unit in Emergency Preparedness and Response

3 UK Health Security Agency, Behavioural Science and Insights Unit

4 King's Centre for Military Health Research and Academic Department of Military Mental Health

5 University College London, Centre for Behaviour Change

6 University College London, Institute of Health Informatics

Corresponding author: Louise E Smith, Post-doctoral Researcher. Department of

Psychological Medicine, King's College London, Weston Education Centre, Cutcombe Road, London, SE5 9RJ. Email: louise.e.smith@kcl.ac.uk. ORCID number: 0000-0002-1277-2564.

Twitter handle: @ louisesmith142 


\section{Highlights}

- Psychological distress has been higher than average throughout the pandemic.

- Distress was higher even after the end of legal restrictions.

- Wellbeing has also been lower than usual.

- Women had greater distress and lower wellbeing than men. 


\begin{abstract}
Psychological distress has been elevated during the COVID-19 pandemic. However, few studies published to date have investigated distress after the first wave of infections (Spring Summer 2020). We investigated distress and wellbeing between April 2020 and September 2021 in England through a series of cross-sectional nationally representative online surveys. People aged 16 years or over living in the UK were eligible for the surveys; for this study we selected only those living in England due to differences in restrictions between UK nations. Distress was measured using the PHQ4 ( $n=46,478$ responses), while wellbeing was measured using the Short Warwick-Edinburgh Mental Wellbeing Scale ( $\mathrm{n}=48,193$ responses). Throughout, approximately $50 \%$ to $60 \%$ of women and $40 \%$ to $50 \%$ of men reported distress, higher than the $20 \%$ to $25 \%$ of men, and $25 \%$ to $30 \%$ of women reported in data. Wellbeing was also worse than population norms, with women reporting lower wellbeing than men. Rates of distress in the English population have been consistently high throughout the pandemic. Patterns of distress have broadly mirrored the pattern of restrictions and case numbers, but there are notable exceptions which indicate that other factors may play a part in population mental health.
\end{abstract}

Keywords: mental health, distress, anxiety, depression, COVID-19, gender 


\section{Introduction}

The COVID-19 pandemic has seen large increases in psychological distress. The pandemic has affected psychological distress in multiple ways. Restrictions on movement and social contact and the imposition of isolation and quarantine have all had negative psychological consequences (Brooks et al., 2020). Fear and anxiety about COVID-19 have also been significant and led to decrements in wellbeing. Other consequences of the pandemic and consequent public health measures can also affect psychological state, including effects via loss of employment, reduced income and disrupted education. These different pathways to distress are highly confounded: restrictions are generally introduced when case numbers and/or perceived threat are high. Restrictions have also fallen at certain periods in the calendar, producing further confounds or interactions with, for example, weather that may impact mental health (Keller et al., 2005).

Psychological distress increased during the early months of the pandemic (Aknin et al., In press). US studies found three or four-fold increases in depression symptoms from before the pandemic to March to April 2020 in nationally representative samples (Ettman et al., 2020; McGinty et al., 2020), with similar results seen in the UK (Fujiwara et al., 2020) and elsewhere (Ebrahimi et al., 2021). Worse wellbeing has also been found during the pandemic (Fujiwara et al., 2020; Helliwell et al., 2021).

There is some evidence that psychological distress mostly returned to pre-pandemic levels by mid-2020 (Aknin et al., In press; Robinson et al., 2021). Fewer studies have investigated psychological distress in late 2020 and even fewer in 2021. In the UK, there is evidence that self-reported anxiety and depression started to rise in September 2020 with a second peak at the end of February 2021 (Fancourt et al., 2021). Estimates suggest that the prevalence of self-reported depressive symptoms in British adults was elevated between June and November 2020 compared to pre-pandemic findings and slightly higher still in January to March 2021 (Office for National Statistics, 2021).

In this study, we used a series of cross-sectional surveys to investigate psychological distress in the English population between April 2020 and September 2021. 


\section{Materials and methods}

\section{$\underline{\text { Design }}$}

Series of online cross-sectional surveys conducted weekly or fortnightly by BMG Research on behalf of the UK Department of Health and Social Care since January 2020. We analysed these as part of the CORSAIR study [the COVID-19 Rapid Survey of Adherence to Interventions and Responses study]; see Smith et al. (2021) for details of methods. For this study, we used data collected between 20 April 2020 and 21 September 2021.

\section{$\underline{\text { Participants }}$}

Participants were recruited from two specialist research panel providers, Respondi $(n=50,000)$ and Savanta $(n=31,500)$. Eligibility criteria for the study were being aged 16 years or over and living in the UK. Quotas were applied based on age and gender (combined) to ensure the sample was broadly representative of the population $(n \approx 2,000$ per wave). Once participants had completed the survey, they were unable to complete the subsequent three survey waves, but they could be invited to participate in the fourth subsequent survey wave onwards.

For this study, we used data collected between 20 April 2020 and 21 September 2021 (wave 13 to 58 ), selecting only participants who reported that they lived in England ( $\approx \approx 1700$ per wave) due to differing restrictions in UK nations. 46,478 responses were included in analyses of distress, while 48,193 responses were included in analyses of wellbeing. Because of how the data were collected, we do not know when responses are from unique or returning individuals.

\section{$\underline{\text { Study materials }}$}

Distress was measured using the PHQ4 (Patient Health Questionnaire), a valid tool for detecting anxiety and depressive disorders (Cronbach's $\alpha=0.92)$ (Kroenke et al., 2009). Higher scores are associated with increased distress. Scores of 0 to 2 are rated "normal." Normative data for the PHQ4 suggest that approximately $75-80 \%$ of men, and $70-75 \%$ of women show no mental distress, i.e. scores of 0 to 2 (UK, German, and Colombian samples: Batty et al., 2016; Kocalevent et al., 2014; Lowe et al., 2010). For both men and women, younger people (e.g. aged 18 to 44 years) show more distress than older people ( 45 years or older) (Batty et al., 2016; Kroenke et al., 2009; Lowe et al., 2010). 
Wellbeing was measured using the Short Warwick-Edinburgh Mental Wellbeing Scale (SWEMWS), another validated tool (Ng Fat et al., 2017; NHS Scotland et al., 2008). Higher scores are associated with better mental wellbeing. Scores for the SWEMWS (7 items) can be transformed to facilitate comparison with the Warwick-Edinburgh Mental Wellbeing Scale (14 items; range 7 to 35; Cronbach's $\alpha=0.90$ ) (Stewart-Brown et al., 2009). Normative data for the SWEMWS in the UK population indicates the (transformed) mean score for women is 23.2 and the mean score for men is 23.7 (Ng Fat et al., 2017).

Measures were not included in all survey waves due to rapidly evolving research questions and space limitations in the questionnaire.

\section{$\underline{\text { Ethics }}$}

This work was conducted as a service evaluation of the Department of Health and Social Care's public communications campaign and, following advice from King's College London Research Ethics Subcommittee, was exempt from ethical approval.

\section{$\underline{\text { Power }}$}

A sample size of 1,700 allows a $95 \%$ confidence interval of plus or minus $2 \%$ for the prevalence estimate for a survey item with a prevalence of around $50 \%$.

\section{$\underline{\text { Analysis }}$}

We charted mental distress and wellbeing during the pandemic (April 2020 to September 2021) for males and females separately. For mental distress, we used the PHQ4, charting the percentage of the sample whose scores indicated distress ( 2 or higher). For wellbeing, we used the SWEMWS, charting mean scores.

\section{Results}

\section{$\underline{\text { Participant characteristics }}$}

Participants (wave 13 to 58) were broadly representative of the English population (53.7\% female [ $\mathrm{n}=42,327 / 78,871]$, mean age 48.2 years [ $\mathrm{SD}=17.7$, range 16 to over 100 years], $82.6 \%$ white British ethnicity [ $\mathrm{n}=65,299 / 79,048 ; 6.7$ white other, $2.4 \%$ mixed, $5.1 \%$ Asian or Asian British, 2.2\% black or black British, 0.5\% Arab or other, 0.6\% prefer not to say]). 


\section{$\underline{\text { Distress }}$}

Distress was higher in women than men throughout the pandemic (Figure 1). The percentage of women showing signs of distress ranged from $49.3 \%$ (95\% CI $46.1 \%$ to $52.5 \%$ ) in data collected 9 to 10 August 2021 to $61.4 \%$ (95\% CI 58.3\% to 64.5\%) from 26 to 27 May 2020. The percentage of men showing signs of distress ranged from $39.6 \%$ (95\% CI $36.2 \%$ to $43.1 \%$ ) from 4 to 6 May 2020 to $48.8 \%$ (95\% CI $45.2 \%$ to $52.4 \%$ ) from 26 to 27 May 2020. 


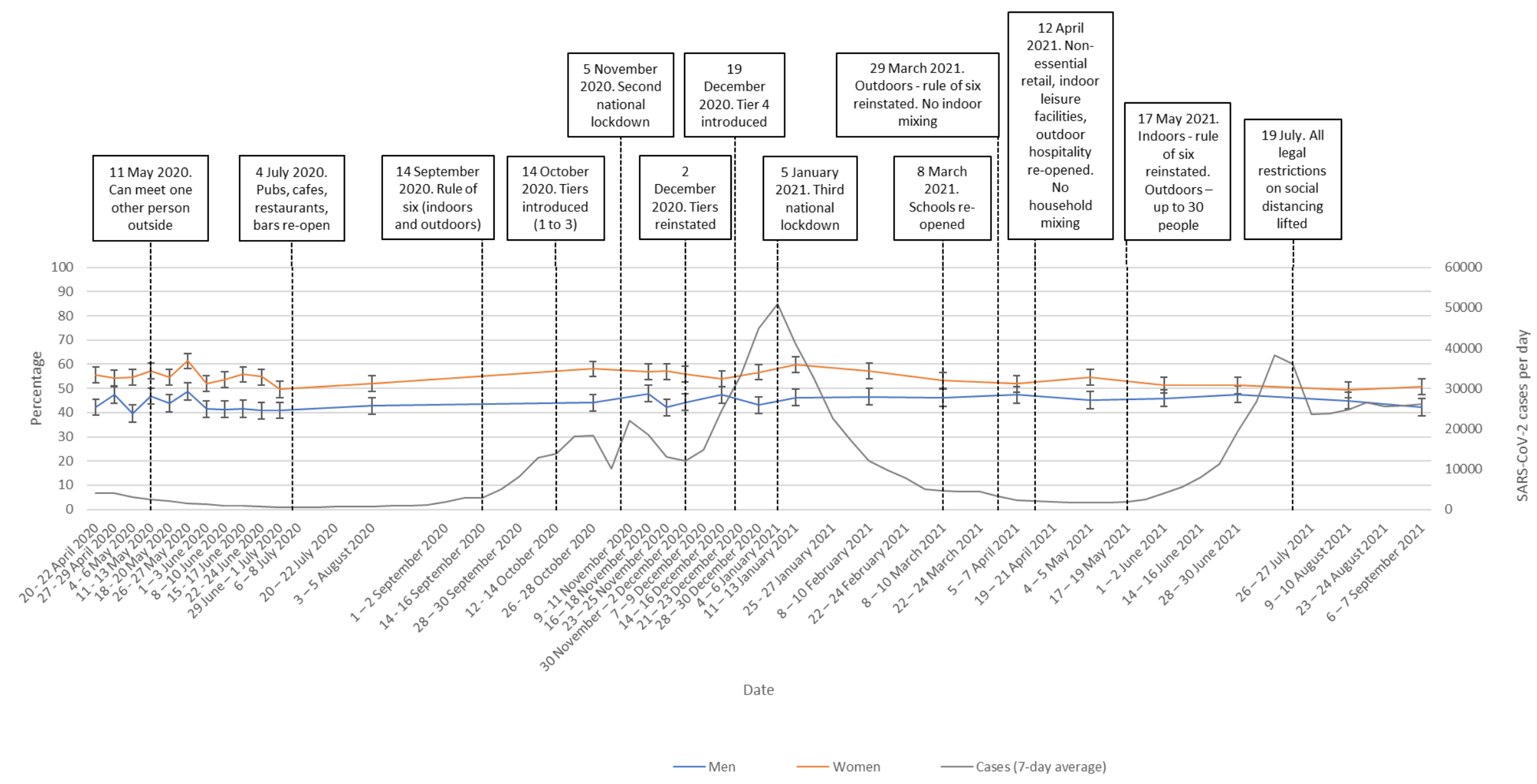

Figure 1. Percentage of participants with signs of increased distress as measured by PHQ4, by gender, with SARS-CoV-2 case numbers for

England.(GOV.UK, 2021) Case rates before June 2020 are an underestimate as only selected people were eligible for testing. Error bars are $95 \%$ confidence intervals. 
Distress was markedly above pre-pandemic norms throughout the period. There was some variation over time, with distress somewhat higher during periods of greater case numbers and stricter restrictions. Distress was highest in the first and third lockdowns (March 2020 and January 2021) and lowest in the summer of 2020. There are also periods where levels of distress were largely constant despite significant changes in case numbers and in restrictions, as in June to September 2021. Men and women showed slightly different patterns of distress, in particular between November 2020 and January 2021, with prevalence increasing in women but decreasing in men.

\section{Wellbeing}

Wellbeing was higher in men than in women throughout the pandemic (Figure 2). Mean transformed wellbeing scores in women ranged between $21.0(\mathrm{SD}=5.1)$ in data collected 22 to 24 February 2021 and $22.4(\mathrm{SD}=4.7) 20$ to 21 September 2021. Mean transformed wellbeing scores in men ranged between $22.0(\mathrm{SD}=5.2)$ in 21 to 23 December 2020 and 23.3 $(\mathrm{SDs}=4.9)$ in 20 to 21 September 2021. 


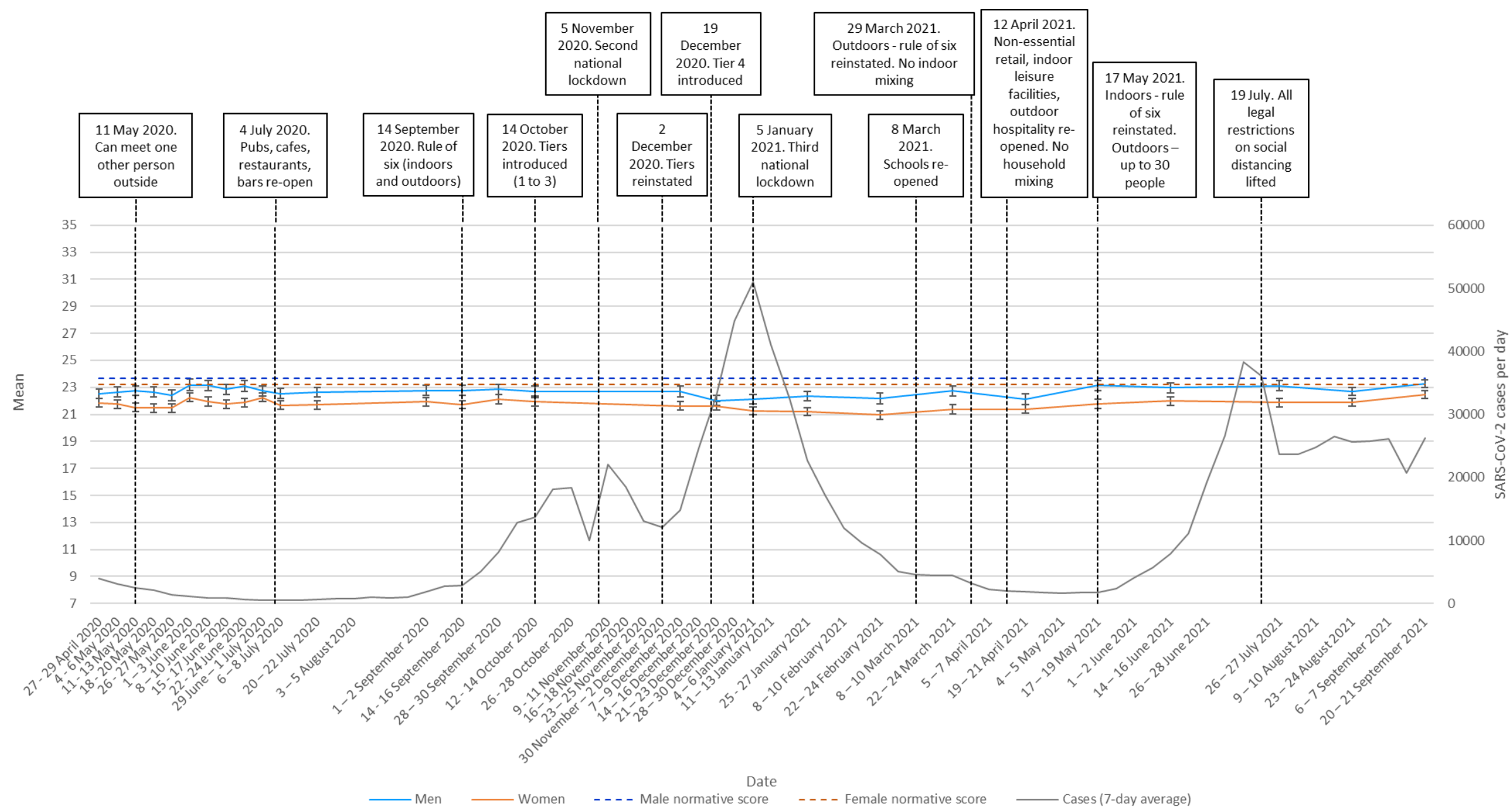

Figure 2. Mean wellbeing scores, by gender, as measured by the SWEMWS (data transformed), with SARS-CoV-2 case numbers for England.(GOV.UK, 2021). Case rates before June 2020 are an underestimate as only selected people were eligible for testing. Error bars are $95 \%$ confidence intervals. 
Wellbeing was below pre-pandemic norms throughout. Wellbeing peaked in both men and women in June 2020, but fell to its lowest between January and February 2021. This dip was particularly noticeable in women. Reductions in wellbeing were greater in the third lockdown than in the first two. Wellbeing increased at the beginning of March 2021, coinciding with the re-opening of schools in England. Between May and July 2021, wellbeing levels in men returned close to their highest point of the pandemic. In women, wellbeing also rose to similar peak levels in mid-June 2021, but subsequently fell. Wellbeing scores did not change with the lifting of all legal restrictions on 19 July.

\section{Discussion}

In England, psychological distress was elevated, and wellbeing lower, during the COVID-19 pandemic compared to population norms. Between April 2020 and September 2021, approximately $50 \%$ to $60 \%$ of women and $40 \%$ to $50 \%$ of men reported distress. This is well over the $25 \%$ indicated by normative data (Batty et al., 2016; Kocalevent et al., 2014; Lowe et al., 2010). Wellbeing was also lower compared to normative scores (Ng Fat et al., 2017). These findings replicate results of other studies conducted earlier in the pandemic indicating that psychological distress has increased during the pandemic in the UK (Fancourt et al., 2021; Pierce et al., 2021). Fewer studies have investigated mental health after the first wave of COVID-19 infections (after summer 2020), but our data are consistent with other studies finding worse mental health (Dale et al., 2021; Vahratian et al., 2021) and life satisfaction (Fancourt et al., 2021) over winter 2020/21, while also extending the study period to September 2021.

While psychological distress was consistently higher, and wellbeing consistently lower, than average, fluctuations in distress and wellbeing were minor. Levels of distress somewhat mirrored case numbers in England and periods of greater restrictions, however we cannot separate the influence of these two factors. There are some notable exceptions where distress and wellbeing did not follow the pattern of restrictions. For example, distress (as measured by PHQ4) increased following the re-opening of hospitality venues on 4 July 2020. Distress also increased in women after legal restrictions were lifted on 19 July 2021. Furthermore, wellbeing peaked in June 2020 while the English population were still under strict lockdown measures. One reason for this could be the warm and sunny weather experienced at that time (Schultz and Tandon, 2020). 
Some politicians and commentators have concluded that mental health problems are a result of lockdowns (e.g., Javid, 2021). However, the literature does not support a simple relationship between lockdowns and population-level psychological distress. In a review of 25 studies published in 2020, Prati and Mancini (2021) found that the psychological impact of COVID-19 lockdowns was small and highly heterogeneous. They reported effects on mental health symptoms generally, and on anxiety and depression, but no significant effect on general distress, positive functioning, social support, loneliness, negative affect or suicidal ideation.

Lockdowns are multifaceted phenomena that may impact wellbeing through different routes. These may not be inevitable consequences of any lockdown but depend on how lockdowns are carried out and what support structures are in place. For example, the greatest increases in mental health problems have been in those who are younger, female, experiencing financial difficulties, and with young children at home (Aknin et al., In press; Vahratian et al., 2021; Yamamoto et al., 2020). While these factors mirror predictors of worse mental health in prepandemic periods (Aknin et al., In press; Dale et al., 2021), they may be further compounded by the effects of lockdowns. For example, our data indicate that women's wellbeing declined with the introduction of the third lockdown (in which schools were shut) and increased following the re-opening of schools in March 2021. The burden of childcare in England during periods of stricter restrictions fell greatly on women (Andrew et al., 2021) and research suggests that time spent engaging in childcare or home-schooling was associated with reduced subjective wellbeing during the pandemic (Aknin et al., In press). There is also an inevitable confound between lockdowns and the pandemic itself: distress may be a direct effect of disease spread, rather than or only because of the restrictions put in place to combat the disease.

Our data indicate that psychological distress was higher, and wellbeing was worse, during the third English lockdown (January to March 2021) than the first lockdown (April to July 2020). This is in line with findings from Austria indicating that mental health was lower during the country's second lockdown (November 2020 to February 2021) compared to the first lockdown (March to April 2020) (Dale et al., 2021). This was thought to be due to a worsening of mental health during the first lockdown, maintenance during the summer while restrictions were eased, and further worsening over the second lockdown. A similar pattern has been found in the UK population, with research finding five distinct trajectories of mental health within the sample between April and October 2020 (very good health throughout, good 
health throughout, recovering [low mental health initially returning to pre-pandemic levels], low mental health throughout, and steadily deteriorating mental health) (Pierce et al., 2021). Strengths of this study include that it investigates psychological distress and wellbeing over a long period of time (April 2020 to September 2021) in a large population. Limitations include that we cannot be certain that the experiences of people who complete online surveys are representative of the general population. It was beyond the scope of this paper to investigate associations with psychological distress.

Rates of psychological distress in the English population have been high and stayed high during the pandemic (April 2020 to September 2021) with only minor fluctuations. Women show greater distress and lower wellbeing than men throughout. While distress and wellbeing broadly follow case numbers in England and periods of greater restrictions, notable instances where releasing of restrictions was not mirrored by an increase in wellbeing suggest that other factors may play a part. 


\section{Funding sources}

This work was funded by the National Institute for Health Research (NIHR) Health Services and Delivery Research programme. Surveys were commissioned and funded by Department of Health and Social Care (DHSC), with the authors providing advice on the question design and selection. LS, RA and GJR are supported by the National Institute for Health Research Health Protection Research Unit (NIHR HPRU) in Emergency Preparedness and Response, a partnership between the UK Health Security Agency, King's College London and the University of East Anglia. RA is also supported by the NIHR HPRU in Behavioural Science and Evaluation, a partnership between the UK Health Security Agency and the University of Bristol. HWWP has received funding from Public Health England and NHS England. NTF is part funded by a grant from the UK Ministry of Defence. The views expressed are those of the authors and not necessarily those of the NIHR, UK Health Security Agency, the Department of Health and Social Care or the Ministry of Defence. The Department of Health and Social Care funded data collection.

\section{Declaration of interest statement}

All authors had financial support from NIHR for the submitted work; RA is an employee of the UK Health Security Agency; HWWP has received additional salary support from Public Health England and NHS England; HWWP receives consultancy fees to his employer from Ipsos MORI and has a PhD student who works at and has fees paid by Astra Zeneca; NTF is a participant of an independent group advising NHS Digital on the release of patient data. All authors are participants of the UK's Scientific Advisory Group for Emergencies or its subgroups.

\section{Author contributions}

Louise E Smith: conceptualisation, data curation, formal analysis, methodology, writing original draft. Richard Amlôt: conceptualisation, funding acquisition, methodology, writing review \& editing. Nicola T Fear: conceptualisation, funding acquisition, methodology, writing - review \& editing. Susan Michie: conceptualisation, funding acquisition, methodology, writing - review \& editing. G James Rubin: conceptualisation, funding acquisition, methodology, writing - review \& editing. Henry WW Potts: conceptualisation, funding acquisition, methodology, writing - original draft. 


\section{References}

Aknin, L., De Neve, J.-E., Dunn, E., Fancourt, D., Goldberg, E., Helliwell, J., Jones, S., Karam, E., Layard, R., Lyubomirsky, S., Rzepa, A., Saxena, S., Thornton, E., VanderWeele, T., Whillans, A., Zaki, J., Karadag Caman, O., Ben Amor, Y., In press. Mental health during the First Year of the COVID-19 pandemic: a review and recommendations for moving forward. Perspectives on Psychological Science.

Andrew, A., Cattan, S., Dias, M.C., Farquharson, C., Kraftman, L., Krutikova, S., Phimister, A., Sevilla, A., 2021. The gendered division of paid and domestic work under lockdown Institute for Fiscal Studies.

Batty, G.D., McIntosh, A.M., Russ, T.C., Deary, I.J., Gale, C.R., 2016. Psychological distress, neuroticism, and cause-specific mortality: early prospective evidence from UK Biobank. J Epidemiol Community Health 70(11), 1136-1139.

Brooks, S.K., Webster, R.K., Smith, L.E., Woodland, L., Wessely, S., Greenberg, N., Rubin, G.J., 2020. The psychological impact of quarantine and how to reduce it: rapid review of the evidence. Lancet 395(10227), 912-920.

Dale, R., Budimir, S., Probst, T., Stippl, P., Pieh, C., 2021. Mental Health during the COVID19 Lockdown over the Christmas Period in Austria and the Effects of Sociodemographic and Lifestyle Factors. Int J Environ Res Public Health 18(7).

Ebrahimi, O.V., Hoffart, A., Johnson, S.U., 2021. Physical Distancing and Mental Health During the COVID-19 Pandemic: Factors Associated With Psychological Symptoms and Adherence to Pandemic Mitigation Strategies. Clinical Psychological Science 9(3), 489-506.

Ettman, C.K., Abdalla, S.M., Cohen, G.H., Sampson, L., Vivier, P.M., Galea, S., 2020.

Prevalence of Depression Symptoms in US Adults Before and During the COVID-19

Pandemic. JAMA Network Open 3(9), e2019686-e2019686.

Fancourt, D., Bu, F., Mak, H.W., Paul, E., Steptoe, A., 2021. Covid-19 Social Study; Results Release 33.

Fujiwara, D., Dolan, P., Lawton, R., Behzadnejad, F., Lagarde, A., Maxwell, C., Peytrignet, S., 2020. The Wellbeing Costs of COVID-19 in the UK. Simetrica-Jacobs, 
GOV.UK, 2021. Cases in England.

https://coronavirus.data.gov.uk/details/cases?areaType=nation\&areaName=England. (Accessed 29 September 2021).

Helliwell, J.F., Huang, H., Wang, S., Norton, M., 2021. Happiness, trust, and deaths under COVID-19, World Happiness Report.

Javid, S., 2021. The economic arguments for opening up Britain are well known. But, for me, the health case is equally compelling, The Mail on Sunday.

Keller, M.C., Fredrickson, B.L., Ybarra, O., Cote, S., Johnson, K., Mikels, J., Conway, A., Wager, T., 2005. A warm heart and a clear head. The contingent effects of weather on mood and cognition. Psychol Sci 16(9), 724-731.

Kocalevent, R.D., Finck, C., Jimenez-Leal, W., Sautier, L., Hinz, A., 2014. Standardization of the Colombian version of the PHQ-4 in the general population. BMC Psychiatry 14, 205.

Kroenke, K., Spitzer, R.L., Williams, J.B., Lowe, B., 2009. An ultra-brief screening scale for anxiety and depression: the PHQ-4. Psychosomatics 50(6), 613-621.

Lowe, B., Wahl, I., Rose, M., Spitzer, C., Glaesmer, H., Wingenfeld, K., Schneider, A., Brahler, E., 2010. A 4-item measure of depression and anxiety: validation and standardization of the Patient Health Questionnaire-4 (PHQ-4) in the general population. J Affect Disord 122(1-2), 86-95.

McGinty, E.E., Presskreischer, R., Han, H., Barry, C.L., 2020. Psychological Distress and Loneliness Reported by US Adults in 2018 and April 2020. JAMA 324(1), 93-94.

Ng Fat, L., Scholes, S., Boniface, S., Mindell, J., Stewart-Brown, S., 2017. Evaluating and establishing national norms for mental wellbeing using the short Warwick-Edinburgh Mental Well-being Scale (SWEMWBS): findings from the Health Survey for England. Qual Life Res 26(5), 1129-1144.

NHS Scotland, University of Warwick, University of Edinburgh, 2008. Short Warwick Edinburgh Mental Wellbeing Scale (SWEMWBS).

Office for National Statistics, 2021. Coronavirus and depression in adults, Great Britain: January to March 2021.

https://www.ons.gov.uk/peoplepopulationandcommunity/wellbeing/articles/coronavirusandde pressioninadultsgreatbritain/latest. (Accessed 5 August 2021). 
Pierce, M., McManus, S., Hope, H., Hotopf, M., Ford, T., Hatch, S.L., John, A., Kontopantelis, E., Webb, R.T., Wessely, S., Abel, K.M., 2021. Mental health responses to the COVID-19 pandemic: a latent class trajectory analysis using longitudinal UK data. Lancet Psychiatry 8(7), 610-619.

Prati, G., Mancini, A.D., 2021. The psychological impact of COVID-19 pandemic lockdowns: a review and meta-analysis of longitudinal studies and natural experiments. Psychol Med 51(2), 201-211.

Robinson, E., Sutin, A.R., Daly, M., Jones, A., 2021. A systematic review and meta-analysis of longitudinal cohort studies comparing mental health before versus during the COVID-19 pandemic. medRxiv, 2021.2003.2004.21252921.

Schultz, A., Tandon, A., 2020. Met Office: Why 2020 saw a record-breaking dry and sunny spring across the UK. https://www.carbonbrief.org/met-office-why-2020-saw-a-recordbreaking-dry-and-sunny-spring-across-the-uk. (Accessed 24 August 2021).

Smith, L.E., Potts, H.W.W., Amlôt, R., Fear, N.T., Michie, S., Rubin, G.J., 2021. Adherence to the test, trace, and isolate system in the UK: results from 37 nationally representative surveys. BMJ 372, n608.

Stewart-Brown, S., Tennant, A., Tennant, R., Platt, S., Parkinson, J., Weich, S., 2009. Internal construct validity of the Warwick-Edinburgh Mental Well-being Scale (WEMWBS): a Rasch analysis using data from the Scottish Health Education Population Survey. Health Qual Life Outcomes 7, 15.

Vahratian, A., Blumberg, S.J., Terlizzi, E.P., Schiller, J.S., 2021. Symptoms of Anxiety or Depressive Disorder and Use of Mental Health Care Among Adults During the COVID-19 Pandemic - United States, August 2020-February 2021. MMWR Morb Mortal Wkly Rep 70(13), 490-494.

Yamamoto, T., Uchiumi, C., Suzuki, N., Yoshimoto, J., Murillo-Rodriguez, E., 2020. The Psychological Impact of 'Mild Lockdown' in Japan during the COVID-19 Pandemic: A Nationwide Survey under a Declared State of Emergency. Int J Environ Res Public Health 17(24). 